\title{
From sleep to arousal to attention deficits in neuropsychiatric disorders
}

\author{
Johannes Thome ${ }^{1} \cdot$ Oliver Tucha ${ }^{2}$
}

Published online: 14 November 2016

(C) Springer-Verlag Wien 2016

Arousal and sleep as two sides of one coin are of considerable neurophysiological and psychological importance. Disturbances of this system may lead or at least contribute to severe neuropsychiatric disorders such as attention-deficit hyperactivity-disorder (ADHD). Thus, the area of arousal and sleep and its impact on attention represents an exciting field for research both in its neurobiological background and its impact on mental health. Sleep disturbances are common in a variety of mental disorders including depression, personality disorders and ADHD. Further, psychotropic substances including psychiatric medication interact with the alertness system and thereby influence individuals' arousal level as well as sleep pattern.

On 5-6 September 2014, the 12th Hansesymposium taking place at the Clinic and Policlinic for Psychiatry and Psychotherapy, University of Rostock, Germany, focused on these very aspects of arousal, sleep and attention and their relevance for psychiatry from a research perspective as well as regarding to clinical practice. Experts in the field presented their up-to-date research results and also contributed to this supplement. In addition, further scientists engaged in this research topic were stimulated to publish their work here. The result is a comprehensive overview of present activities aiming at elucidating physiological mechanisms underlying sleep and arousal and pathogenetic

Oliver Tucha

o.m.tucha@rug.nl

1 Department of Psychiatry, University of Rostock, Rostock, Germany

2 Department of Clinical and Developmental Neuropsychology, University of Groningen, Groningen, The Netherlands alterations which may contribute to specific symptoms of neuropsychiatric disorders.

The effects of prefrontal rTMS on autonomic reactions to affective pictures have been investigated by Berger et al.

Electronic media use and insomnia complaints are the focus of the work by Cohrs et al. which specifically focuses on gender differences.

Den Heijer et al. systematically review the literature regarding the effects of physical exercise on cognition and behaviour in children with ADHD.

An update on the role of sleep problems and circadian clock genes in ADHD and mood disorders during childhood and adolescence is given by Dueck et al.

Fuermaier et al. discuss the issue of driving and ADHD.

Goerke et al. present a pilot study finding that successful physical exercise-induced weight loss in the elderly is modulated by habitual sleep duration, and discuss in a second paper the implications of sleep-dependent memory consolidation for psychiatry.

Groen et al. present data about the influence of stimulant medication on blink rate and time in ADHD patients.

Koo et al. analyse current source density of resting-state EEG in depressed patients.

Krysta et al. discuss sleep and inflammatory markers in various psychiatric disorders and also present a second paper on cognitive deficits in adults with obstructive sleep apnea compared to children and adolescents.

Marx et al. studied serum fatty acid composition in geriatric depression.

The impact of aging on the circadian clock is reviewed by Popa-Wagner et al.

Skarupke et al. investigate insomnia complaints and substance use in adolescents based on data resulting from the KiGGS study (German Health Interview and Examination Survey for Children and Adolescents). 
Tucha et al. investigate sustained attention in adult ADHD focusing on time-on-task effects.

Weinhold et al. present a study based on sleep recordings in individuals with borderline personality disorder before and after trauma therapy.
In summary, we hope that this supplement will reflect the wide range of scientific efforts in this exciting field and inspires all those who work either in basic research about the phenomena of sleep and arousal or in clinical research with patients affected by alterations in this system, or plan to do so. 